\title{
Review Article \\ Effect of Neoadjuvant Chemoradiotherapy with Capecitabine versus Fluorouracil for Locally Advanced Rectal Cancer: A Meta-Analysis
}

\author{
Guo-Chen Liu, ${ }^{1}$ Jun-Ping Yan, ${ }^{2}$ Qing He, ${ }^{3}$ Xin An, ${ }^{4}$ Zhi-Zhong Pan, ${ }^{5}$ and Pei-Rong Ding ${ }^{5}$ \\ ${ }^{1}$ Department of Gynecologic Oncology, Sun Yat-sen University Cancer Center, State Key Laboratory of Oncology in South China, \\ Collaborative Innovation Center for Cancer Medicine, Guangdong 510060, China \\ ${ }^{2}$ Department of Laboratory Medicine, Guangdong No. 2 Provincial People's Hospital, Guangdong, China \\ ${ }^{3}$ Department of General Surgery, People’s Hospital of Yuxi City, Yunnan, China \\ ${ }^{4}$ Department of Medical Oncology, Sun Yat-sen University Cancer Center, State Key Laboratory of Oncology in South China, \\ Guangdong, China \\ ${ }^{5}$ Department of Colorectal Surgery, Sun Yat-sen University Cancer Center, State Key Laboratory of Oncology in South China, \\ Guangdong 510060, China
}

Correspondence should be addressed to Zhi-Zhong Pan; panzhzh@sysucc.org.cn and Pei-Rong Ding; 463701222@qq.com

Received 2 April 2016; Accepted 30 June 2016

Academic Editor: Carlos Fernandez-Martos

Copyright (C) 2016 Guo-Chen Liu et al. This is an open access article distributed under the Creative Commons Attribution License, which permits unrestricted use, distribution, and reproduction in any medium, provided the original work is properly cited.

\begin{abstract}
A meta-analysis was carried out to compare the efficacy and safety of capecitabine plus radiation with 5-fluorouracil (5-FU) plus radiotherapy (RT) as neoadjuvant treatment in locally advanced rectal cancer (LARC). We searched the Cochrane database, Ovid, Medline, Embase, ISI databases, and Chinese Biomedical Literature Database between January 1998 and October 2014. Trials of capecitabine compared with 5-FU plus RT as neoadjuvant treatment for LARC were considered for inclusion. RevMan software was used to analyze these data. Nine trials were included in this meta-analysis, which covered a total of 3141 patients. The metaanalysis showed that capecitabine group had statistically significant better pCR rates (OR, 1.34; 95\% CI, 1.10-1.64; $P=0.003)$, T downstaging rates $(\mathrm{OR}, 1.58 ; 95 \% \mathrm{CI}, 1.22-2.06 ; P=0.0007)$, $\mathrm{N}$ downstaging rates (OR, 2.06; 95\% CI, 1.34-3.16; $P=0.001)$, less distant metastasis (OR, 0.63; 95\% CI, 0.44-0.88; $P=0.007)$, and lowered leucocytes (OR, 0.25; 95\% CI, 0.11-0.54; $P=0.0005)$, but with higher incidence of hand-foot syndrome (HFS) $(\mathrm{OR}, 4.43 ; 95 \% \mathrm{CI}, 1.59-12.33 ; P=0.004)$. Capecitabine was more efficient than 5 -FU in terms of tumor response in neoadjuvant treatment for patients with LARC and favourably low toxicity with the exception of HFS.
\end{abstract}

\section{Introduction}

Neoadjuvant chemoradiotherapy with fluoropyrimidine is the standard treatment for locally advanced rectal cancer (LARC), as supported by results of the randomized phase III study conducted by the German Rectal Cancer Study Group [1]. 5-FU combined with leucovorin (LV) is the most commonly administered concurrent chemotherapy. Modifications of perioperative fluorouracil treatment have been investigated in many Phase II and Phase III trials in an attempt to improve overall survival and disease-free survival rate. Compared to bolus 5-FU and pelvic radiotherapy (RT), patients who received concurrent RT with protracted 5-FU infusion had an improved period of time to relapse and postoperative survival in both preoperative [2-4] and postoperative chemoradiotherapy $[5,6]$. Therefore, protracted infusion 5 -FU is accepted as the standard regimen for concurrent chemoradiotherapy for rectal cancer at many institutions. However, protracted 5-FU infusion requires an indwelling venous catheter, which is associated with increased complications (infection, bleeding, thrombosis, and pneumothorax) [7] and patients' discomfort.

Capecitabine, an orally administered fluoropyrimidine, was shown to have antitumor activity in metastatic colorectal cancer, and it is also more convenient to use [8]. In several randomized trials, capecitabine had been shown to be at least 
equivalent in efficacy to 5-FU in metastatic colorectal cancer (mCRC) [9, 10]. Capecitabine is preferentially converted to 5-FU in tumor tissue through a three-step enzymatic pathway with the participation of thymidine phosphorylase. $\mathrm{X}$-ray irradiation was found to induce the synthesis of thymidine phosphorylase, providing a rationale that the usage of capecitabine combined with RT might be associated with an improved therapeutic index in patients with cancer [11].

Up to now, only two randomized trials $[12,13]$ and several retrospective studies [14-20] had compared capecitabine versus 5-FU/LV in neoadjuvant chemoradiotherapy regimens for patients with LARC; however, in terms of pathologic complete response ( $\mathrm{pCR}$ ) rate and toxicities, the results of these studies were not consistent. Therefore, we carried out a meta-analysis to determine the difference in efficacies and toxicities of these two regimens when used as treatment combination with RT in patients with LARC.

\section{Methods}

2.1. Literature Search. The Cochrane database, Ovid, Medline, Embase, ISI databases, and Chinese Biomedical Literature Database were searched from January 1998 up to October 2008. The following Mesh search headings were used: "rectal neoplasms", "neo-adjuvant therapy", "chemoradiotherapy", "capecitabine", and "fluorouracil". All relevant Oncology Meetings Proceedings (ASCO, AACR, and ASCO GI) and bibliographies of references and reviews were also identified. Furthermore, we searched the reference lists of retrieved relevant articles in order to broaden the scope, and all abstracts, studies, and citations scanned were reviewed too. There were no language restrictions.

2.2. Inclusion and Exclusion Criteria. Trials meetings which have the following two criteria were included: (1) compared capecitabine to 5-FU (with or without LV) as neoadjuvant treatment for LARC and (2) illustrated at least one of the outcome measures: tumor response rate, sphincter preservation rate, or adverse effects of treatment/toxicity. When two studies were reported by the same institution, either the one with better quality or the most recent publication was included in this analysis.

Studies were excluded from the analysis if the outcomes of interest were not reported or it was impossible to calculate these from the published results.

2.3. Data Extraction. Two researchers assessed all abstracts identified by the above search strategies for subject relevance, respectively. The full publications of all possibly relevant abstracts were obtained and formally assessed for inclusion. Two reviewers independently extracted the following information from each included study: first author, year of publication, characteristics of study population, study design, pathological response to chemoradiation, sphincter preservation, and adverse effects. Adverse effects of treatment/toxicities (grade 3 or 4 ) data were collected according to National Cancer Institute Common Toxicity Criteria (NCICTC v4.0).
2.4. Statistical Methods. Statistical analyses were carried out using Review Manager Version 5.0 software (Nordic Cochrane Centre, Copenhagen, Denmark). We calculated the odds ratio (OR) for dichotomous data with $95 \%$ confidence intervals (CIs) for all analyses. Two techniques were used to calculate the pooled OR estimates: the Mantel-Haenszel method [21], which assumes a fixed-effects model, and the DerSimonian-Laird method [22], which assumes a randomeffects model. If there was no heterogeneity, the fixedand the random-effects models provided similar results, and we chose fixed-effects model. When heterogeneity was found, the random-effects model was considered to be more appropriate, although both models might be biased. An estimation of disease-free survival based on Kaplan-Meier plots was performed by DigitizeIt software 2.06 (Bormann, bormisoft@digitizeit.de).

Statistical heterogeneity between studies was assessed by the chi-squared test with significance set at a $P$ value of 0.10 and the quantity of heterogeneity measured using the $I^{2}$ statistic. Negative values of $I^{2}$ were put equal to zero, so that $I^{2}$ lay between $0 \%$ and $100 \%$. A value of $0 \%$ indicates no observed heterogeneity, and larger values show increasing heterogeneity. Publication bias was assessed by use of a funnel plot [23].

\section{Results}

3.1. Description of Studies. Figure 1 showed the process used to select potentially relevant studies for inclusion; 114 records were identified by the primary computerized literature search. However, after screening the titles and abstracts, we excluded 95 studies which were either case series, review articles $(n=40)$, or irrelevant to the current study $(n=55)$. The full texts of the retrieved 19 manuscripts were clearly read, and the reference lists were checked. After excluding 4 articles including other regimens and 6 studies which did not include both 5-FU and capecitabine simultaneously, finally, we included the remaining 9 trials in the meta-analysis [1220], having covered a total of 3141 patients. Among these patients, 1541 pts were found to be treated with capecitabine and 1600 pts with 5-FU as neoadjuvant chemotherapy regimen. It should be noted that one trial [13] alone out of the 9 trials contributed almost one-half of the patients included in the present meta-analysis. The characteristics of the nine studies included in this paper were shown in Table 1, two of them were prospective studies $[12,13]$ with a combined total of 2000 subjects, and 7 were retrospective studies which covered a total of 1141 patients [14-20].

It should be noted that the NSABP-R-04 protocol [13] was a two-step study, (1) from September 2004 to October 2005, 293 patients were randomly assigned in the two-arm portion of the study (capecitabine versus 5-FU) and (2) from October 2005 to August 2010, additional 1315 patients were randomly assigned into four-arm study: 330 patients to RT plus 5-FU, 329 patients to RT plus 5-FU and oxaliplatin, 326 patients to RT plus capecitabine, and 330 patients to RT plus capecitabine and oxaliplatin. The author did not mention the pCR rates of patients received capecitabine/5FU monotherapy plus RT. However, in this protocol, there 


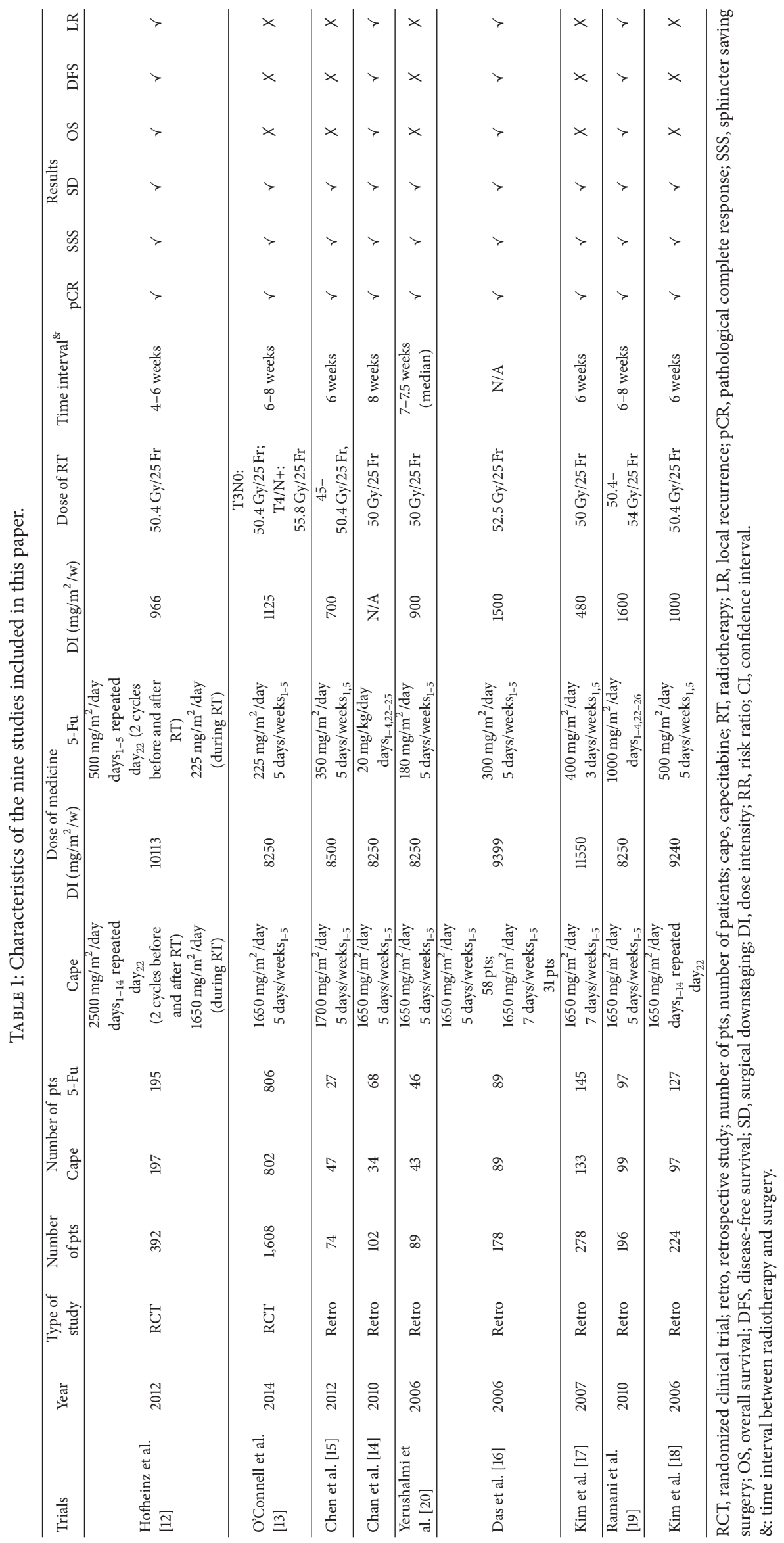




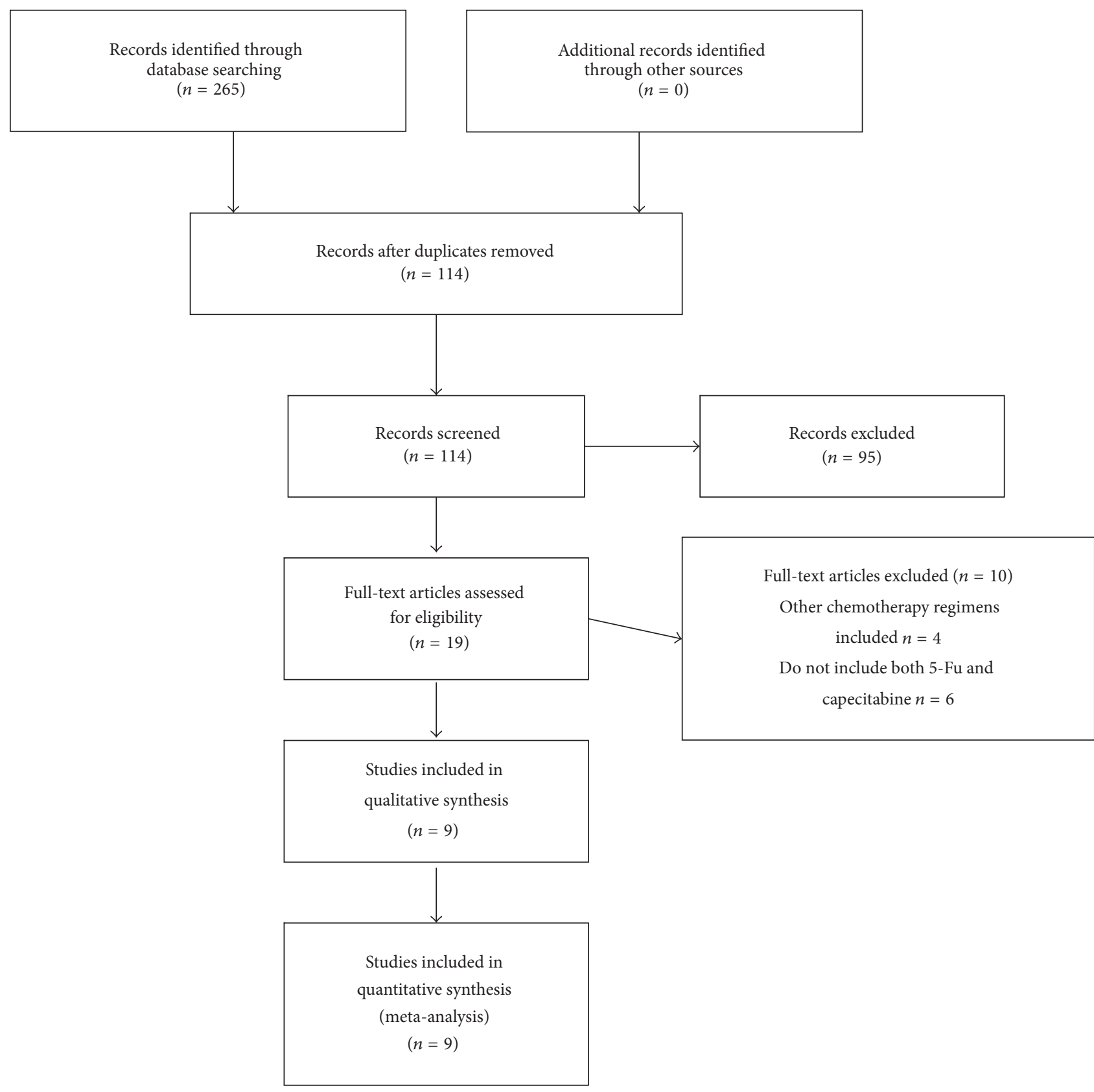

FIGURE 1: The Quality of Reporting of Meta-Analyses (QUOROM) statement flow diagram.

was no significant detrimental effect on pCR rates when neoadjuvant chemotherapy was combined with oxaliplatin or not $(P=0.42)$. Therefore, all 1608 patients were included in the analysis for $\mathrm{pCR}$ rates.

3.2. Tumor Response. Tumor response rates were reported in all trials. All 9 trials reported pCR rates (Figure 2); the fixed-effect pooled estimate including 2785 patients evaluated for OR showed a statistically significant increased $O R$ for capecitabine (OR, 1.34; 95\% CI, 1.10-1.64; $P=0.003)$. The inclusion of the two RCTs only to this analysis led to an RR of 1.20 (95\% CI, 0.98-1.47; $P=0.07$ ) (Figure 3). Seven trials $[12-15,18-20]$ reported $\mathrm{T}$ downstaging (the reduction of the $\mathrm{T}$ stage by at least one level after neoadjuvant chemoradiation); random-effect pooled estimate demonstrated an increased OR for capecitabine (OR, 1.58; 95\% CI, 1.22-2.06; $P=0.0007)$ (Figure 4). However, there were heterogeneities between these trials $(I=53 \%, P=0.05)$. Four trials $[12,15,18,19]$ reported $\mathrm{N}$ downstaging (the reduction of the $\mathrm{N}$ stage by at least one level after neoadjuvant chemoradiation); randomeffect pooled estimate demonstrated an increased OR for capecitabine as well (OR, 2.06; 95\% CI, 1.34-3.16; $P=0.001)$ (Figure 5).

3.3. Type of Surgical Resection. Data on the rate of sphinctersparing resection were available for all the 9 trials; no 
Review: capecitabine versus 5-FU

Comparison: 01 capecitabine versus 5-FU

Outcome: 01 pCR rates of capecitabine or 5-FU

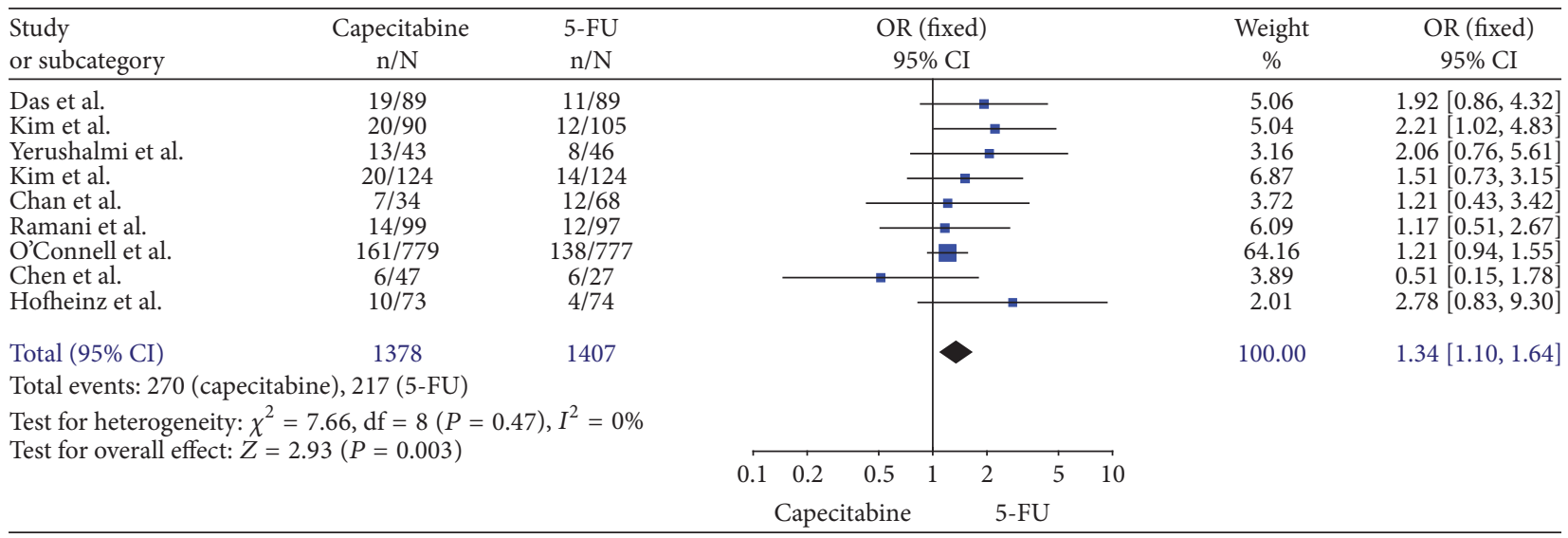

FIGURE 2: Forest plot of odds ratio (OR) for pCR rate of all trials.

Review: capecitabine versus 5-FU

Comparison: 01 capecitabine versus $5-\mathrm{FU}$

Outcome: 02 RCT

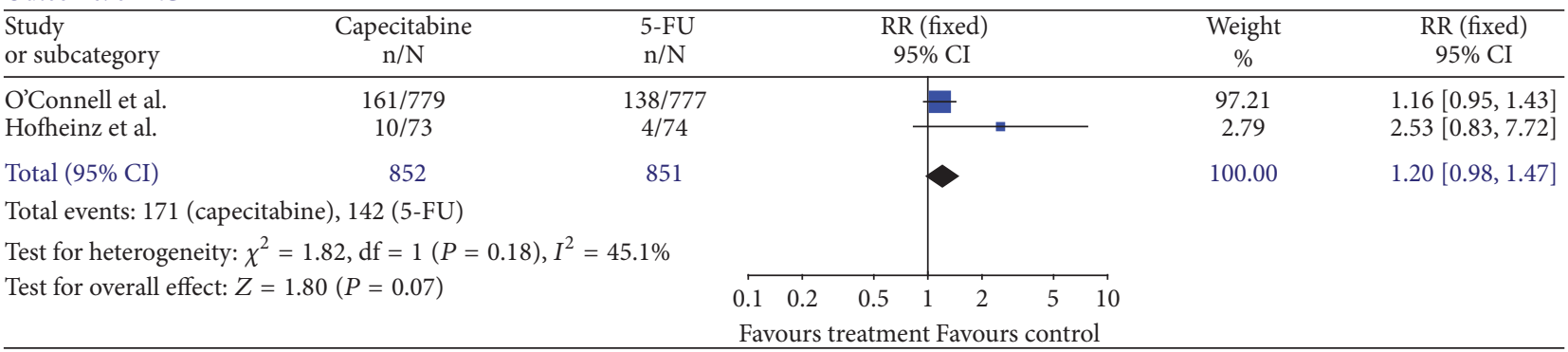

FIGURE 3: Forest plot of relative risk (RR) for pCR rate of RCTs only.

Review: capecitabine versus 5-FU

Comparison: $08 \mathrm{~T}$ downstaging

Outcome: $01 \mathrm{~T}$ downstaging

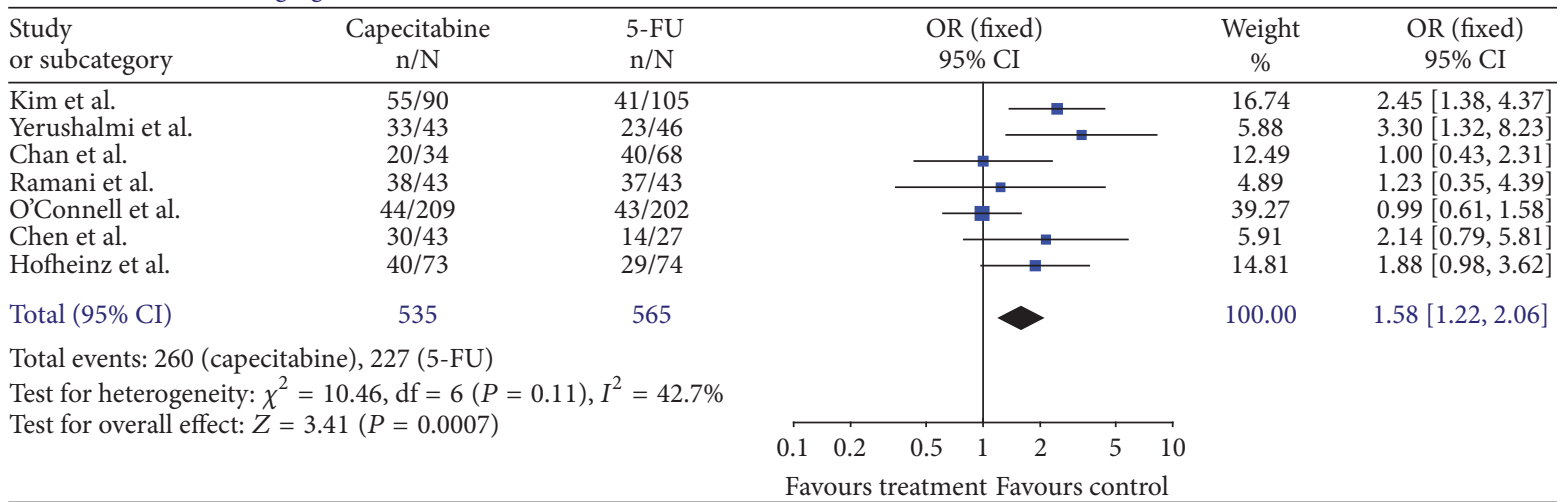

FIgURE 4: Forest plot of odds ratio (OR) for T downstaging rate.

statistically significant difference was demonstrated between the two regimens $(\mathrm{OR}, 1.09 ; 95 \% \mathrm{CI}, 0.92-1.28 ; P=0.31)$ (Figure 6).

3.4. Recurrence. Four trials $[12,14,16,19]$ provided data for disease-free survival; however, Kaplan-Meier plots of disease-free survival were only available in 3 trials $[12,14,19]$, including 690 patients (capecitabine, $n=330 ; 5-\mathrm{FU}, n=360$ ). The meta-analysis of the pooled data demonstrated that the 3 -year disease-free survival was in favour of capecitabinebased regimens (HR, 0.65; 95\% CI, 0.44-0.96, $P=0.03$; Figure 7$) .16 .2 \%(68 / 419)$ patients in capecitabine group and 
Review: capecitabine versus 5-FU Comparison: $09 \mathrm{~N}$ downstaging Outcome: $01 \mathrm{~N}$ downstaging

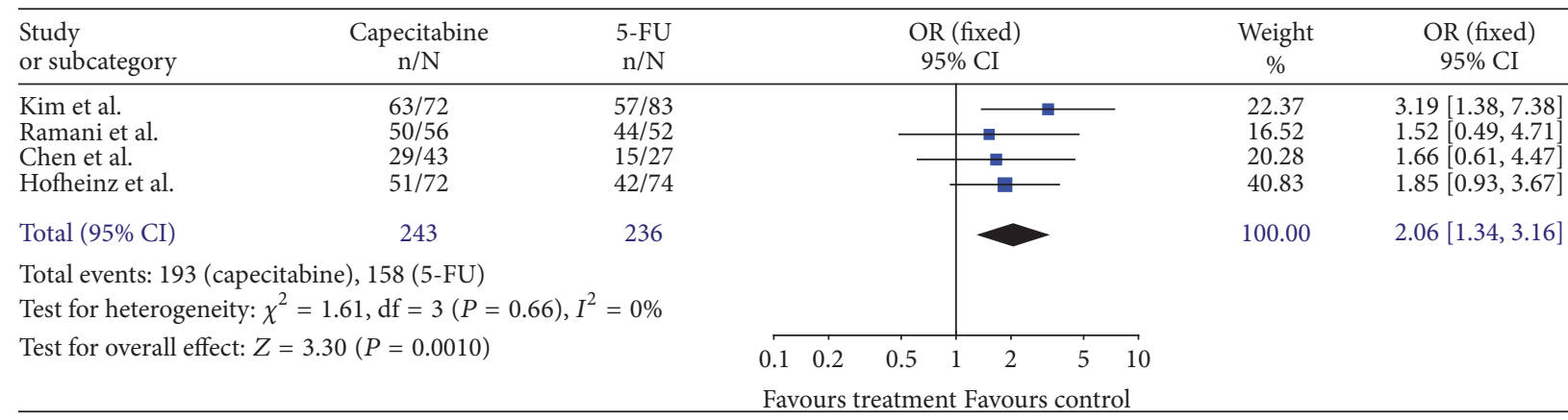

FIGURE 5: Forest plot of odds ratio (OR) for $\mathrm{N}$ downstaging rate.

Review: capecitabine versus 5-FU

Comparison: 05 surgery

Outcome: 01 Sphincter saving versus sphincter not saving

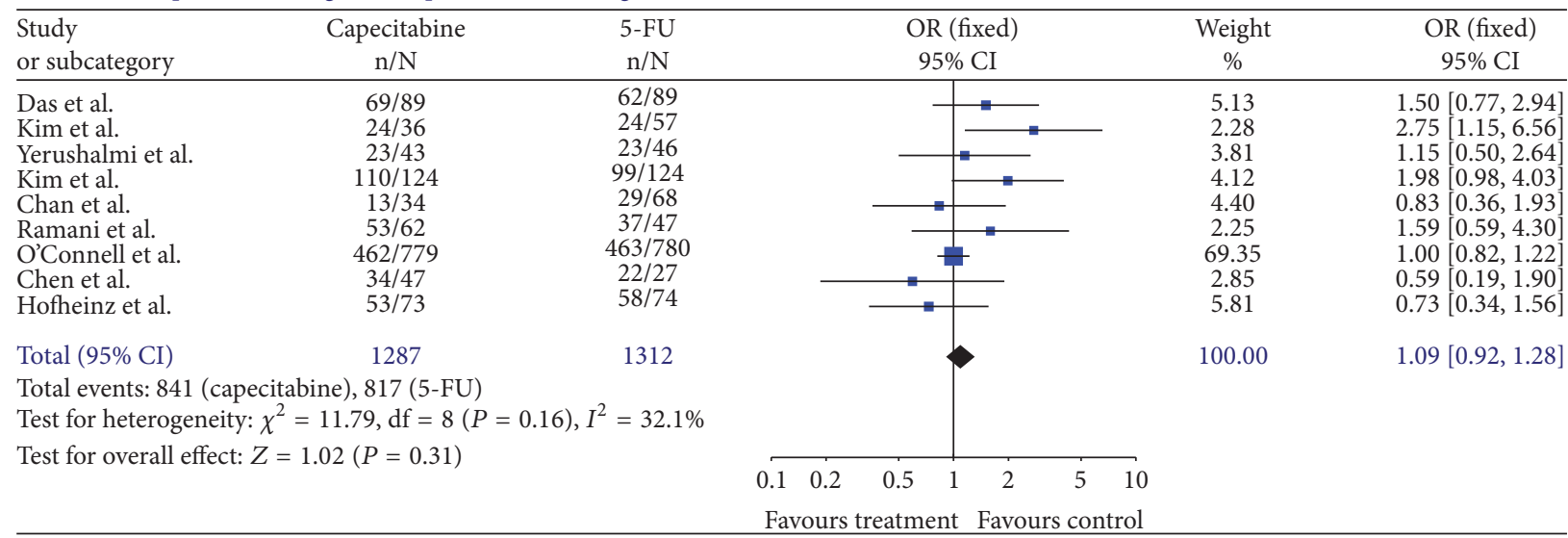

FIGURE 6: Forest plot of odds ratio (OR) for sphincter-sparing resection rate.

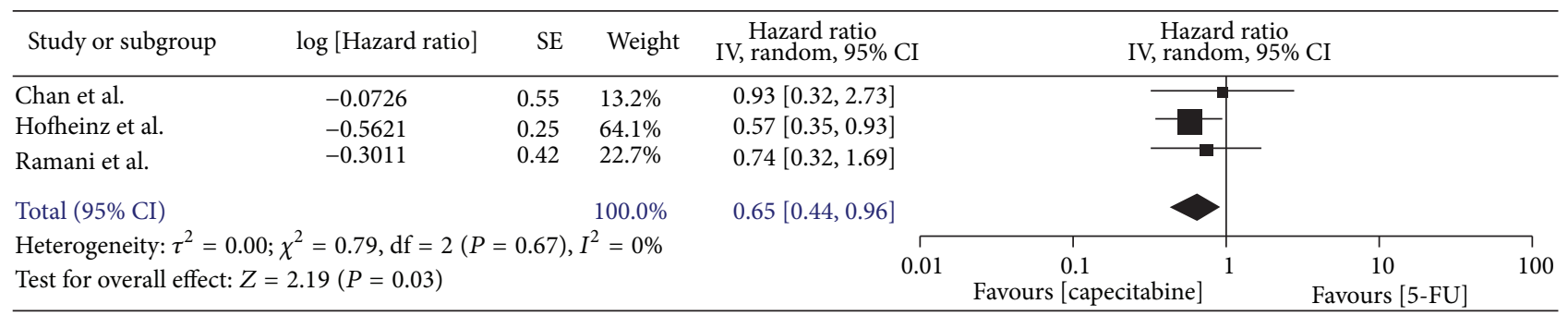

FIGURE 7: Forest plot of hazard ratio (HR) for 3-year disease-free survival.

23.8\% (107/449) in 5-FU group had distant recurrence, and there was significant difference (OR, 0.63; 95\% CI, 0.44$0.88 ; P=0.007$, Figure 8$)$. However, there was no significant difference in the local recurrence rate between the two groups (OR, 0.88; 95\% CI, 0.47-1.65; $P=0.69$, Figure 9).

3.5. Toxicity. With regard to toxicity, we chose the most frequently reported side-effects (at least mentioned in four trials) to obtain reliable comparisons between capecitabine and 5-FU regimen. Thus, toxicity was not evaluated completely and the reported results must be interpreted with caution.
The adverse events included lowered hemoglobin, lowered leucocytes, lowered platelets, nausea, vomiting, diarrhea, mucositis, hand-foot syndrome, and radiation dermatitis. The most frequent toxicity was diarrhea in patients receiving either capecitabine $(87 / 1204)$ or 5-FU (95/1252) combined with RT (Table 2).

Grade 3 or worse lowered leucocytes (reported by 7 trials $[12,14-19])$ were significantly less prominent in patients receiving capecitabine and $\mathrm{RT}(\mathrm{OR}, 0.25$; 95\% CI, 0.11-0.54; $P=0.0005)$ (Figure 10). Moreover, after excluding the only trial [17] in which capecitabine group had more patients with 
Review: capecitabine versus 5-FU

Comparison: 10 metastasis

Outcome: 01 metastasis

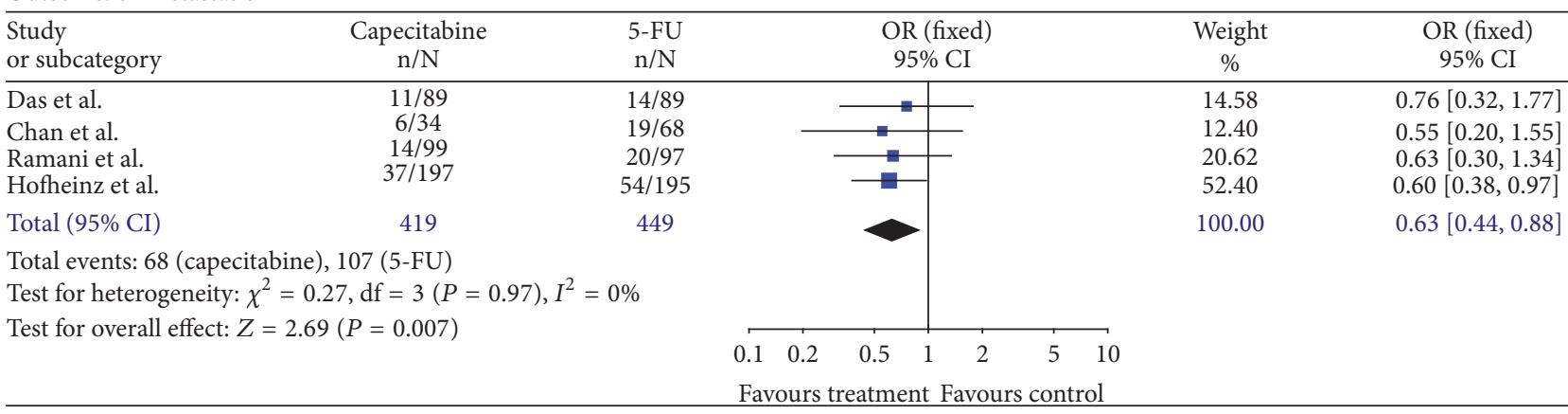

FIGURE 8: Forest plot of odds ratio (OR) for metastasis rate.

Review: capecitabine versus 5-FU

Comparison: 06 local recurrence

Outcome: 01 local recurrence

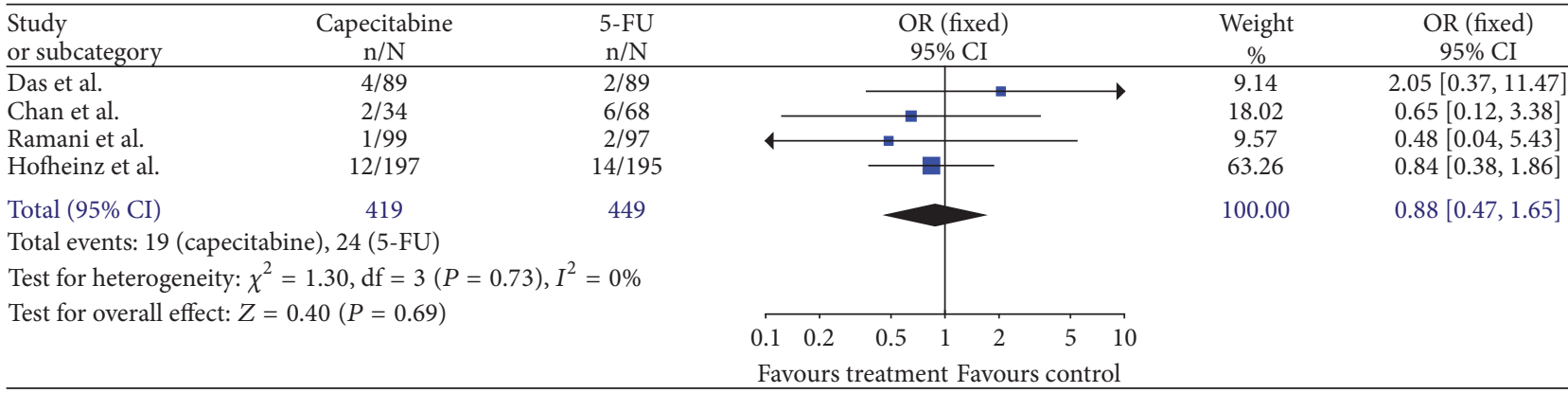

FIGURE 9: Forest plot of odds ratio (OR) for local recurrence rate.

Review: capecitabine versus 5-FU

Comparison: 04 detailed toxicity

Outcome: 02 lowered leucocytes

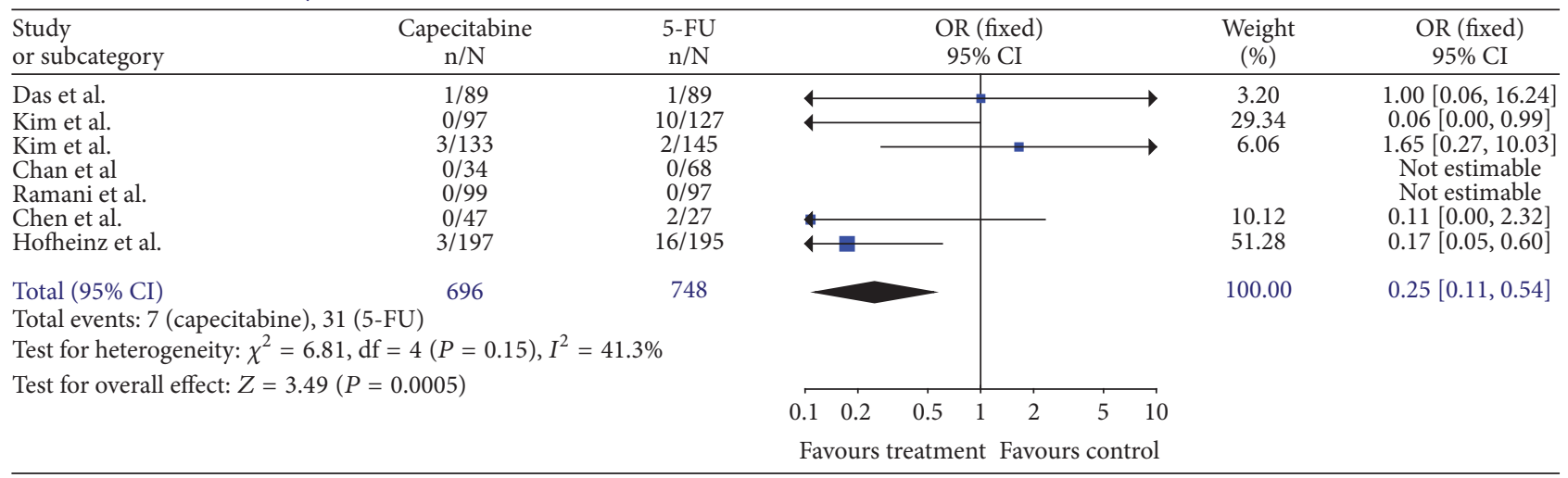

FIGURE 10: Forest plot of odds ratio (OR) for lowered leucocytes rate.

lowered leucocytes, the difference was more obvious (OR $0.08 ; 95 \% \mathrm{CI}, 0.02$ to $0.30 ; P=0.0002$ ) with less heterogeneity $(P=0.48)$. However, the frequency of hand-foot syndrome (reported by all trials) appeared higher in the capecitabine and RT group (OR, 4.43; 95\% CI, 1.59-12.33; $P=0.004$ ) (Figure 11). A sensitivity analysis showed that several adverse effects were not statistically different between capecitabine and 5-FU, such as lowered hemoglobin [12, 14-19], lowered platelets $[12,16-20]$, nausea $[12-15,18,19]$, vomiting $[12,13,15$, $18,19]$, mucositis $[12,14,16,19]$, and radiation dermatitis [12$16,18,20]$, whereas, with respect to severe diarrhea (reported by 7 trials [12-20]), the findings of major heterogeneity between these trials $(I=64.5 \%$ and $P=0.004)$ restricted a valid interpretation of the pooled estimates. 
TABLE 2: Toxic (grade 3 or worse* ${ }^{*}$ effects in trials comparing capecitabine with 5-Fu.

\begin{tabular}{|c|c|c|c|c|c|c|c|}
\hline \multirow{2}{*}{ Toxicity } & \multirow{2}{*}{ Number of trials } & \multicolumn{2}{|c|}{ Number of cases } & \multirow{2}{*}{ OR (95\% CI) } & \multicolumn{2}{|c|}{ Test of homogeneity } & \multirow{2}{*}{$P$ value } \\
\hline & & Cape & $5-\mathrm{Fu}$ & & $I^{2}(\%)$ & $P$ value & \\
\hline Lowered hemoglobin & 7 & $6 / 696$ & $5 / 748$ & $1.13(0.36,3.54)^{\#}$ & 24.6 & 0.27 & 0.83 \\
\hline Lowered leucocytes & 7 & $7 / 696$ & $31 / 748$ & $0.24(0.11,0.54)^{\#}$ & 28.9 & 0.23 & 0.0005 \\
\hline Lowered platelets & 6 & $0 / 658$ & $4 / 699$ & $0.30(0.05,1.88)^{\#}$ & 0 & 0.99 & 0.2 \\
\hline Nausea & 6 & $10 / 906$ & $4 / 905$ & $2.30(0.79,6.70)^{\#}$ & 0 & 0.51 & 0.13 \\
\hline Vomiting & 6 & $7 / 1038$ & $2 / 1049$ & $3.04(0.72,12.75)^{\#}$ & 0 & 0.66 & 0.13 \\
\hline Diarrhea & 9 & $87 / 1204$ & $95 / 1252$ & $0.92(0.67,1.24)^{\S}$ & 66.7 & 0.002 & 0.57 \\
\hline Mucositis & 4 & $1 / 419$ & $4 / 449$ & $0.33(0.05,2.10)^{\#}$ & 0 & 0.64 & 0.24 \\
\hline Hand-foot syndrome & 9 & $17 / 1204$ & $3 / 1252$ & $4.43(1.59,12.33)^{\#}$ & 0 & 0.48 & 0.004 \\
\hline Radiation dermatitis & 7 & $26 / 972$ & $32 / 1010$ & $0.85(0.51,1.44)^{\S}$ & 49.3 & 0.08 & 0.55 \\
\hline
\end{tabular}

$\mathrm{RR}$, risk ratio; cape, capecitabine; $\mathrm{CI}$, confidence interval.

${ }^{\#}$ Fixed-effect model.

${ }^{\mathfrak{S}}$ Random-effects model.

${ }^{*}$ National Cancer Institute Common Terminology Criteria for Adverse Events, version 4.0.

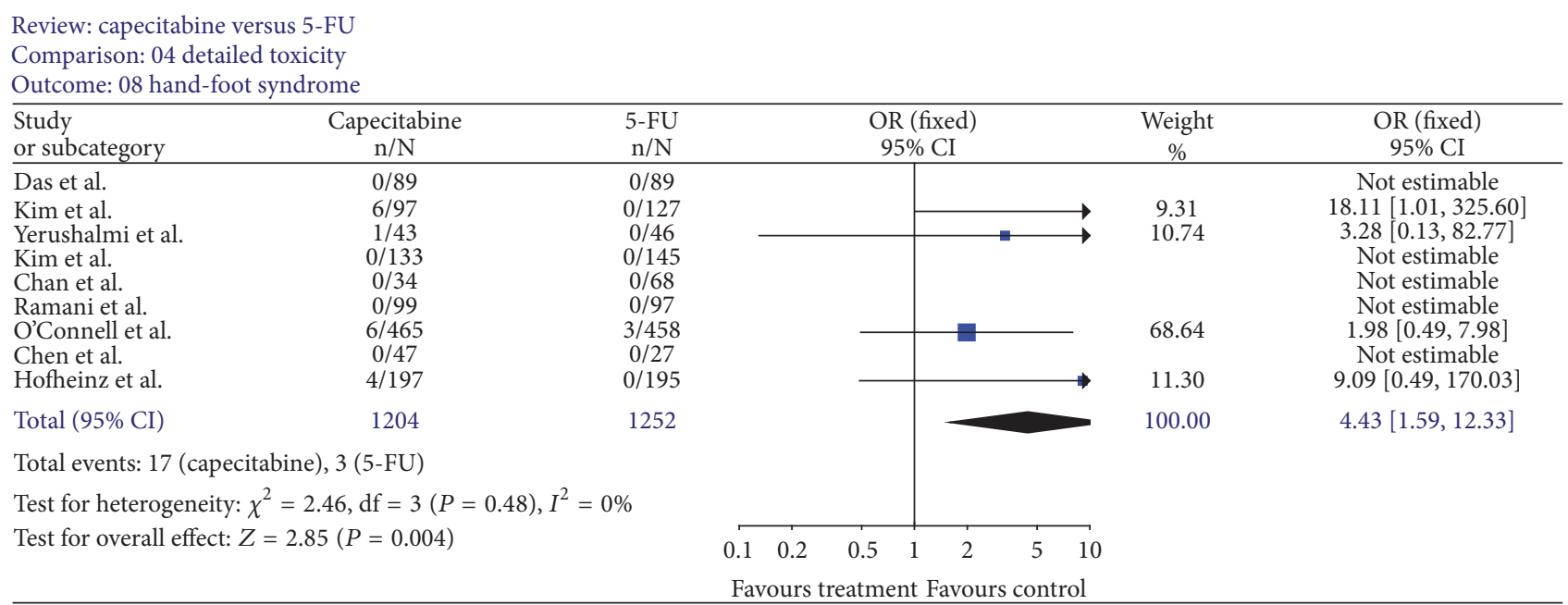

FIGURE 11: Forest plot of odds ratio (OR) for hand-foot syndrome rate.

3.6. Evaluation of Publication Bias. A funnel plot of the studies used in the meta-analysis reporting on capecitabine versus 5-FU as chemotherapy plus RT used as neoadjuvant treatment for patients with LARC was shown in Figure 12. There was no evidence of publication bias (all studies were equally distributed round the vertical axis).

\section{Discussion}

This meta-analysis compared the efficacy and safety of capecitabine plus radiation with 5-FU plus RT as neoadjuvant treatment in LARC. In the light of its favourable efficacy and low toxicity, the analysis showed that capecitabine was superior to 5-FU in neoadjuvant treatment for patients with LARC. Capecitabine plus RT might be regarded as standard neoadjuvant treatment in patients with LARC considering its advantage.

The previous work by Meta-Analysis Group in Cancer proved continuous intravenous infusion (CIV) 5-FU is superior to bolus 5-FU in terms of tumor response based on other

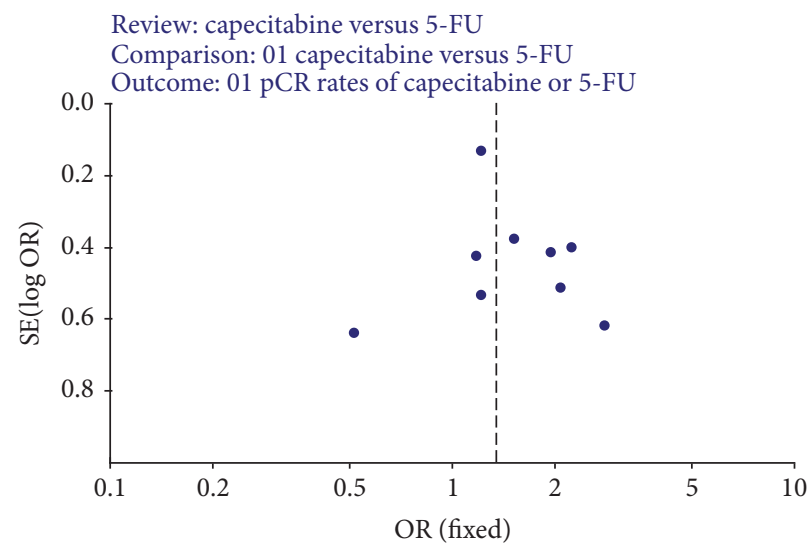

FIGURE 12: Funnel plot of studies included in this meta-analysis.

studies of advanced colorectal cancer [24]. Capecitabine mimics CIV 5-FU in its pharmacologic action in vivo; it has shown equivalent efficacy to CIV 5-FU plus leucovorin on 
metastatic colorectal cancer, but without the use of venous catheter and better patient tolerability. Both the two included RCT trials confirmed the equivalence of CIV 5-FU and capecitabine in neoadjuvant chemoradiotherapy regimens for patients with LARC. However, regarding outcome and toxicity evaluation, heterogeneities still remained between different studies.

This meta-analysis brought together all currently available data comparing capecitabine with 5-FU plus RT used as neoadjuvant treatment for patients with LARC.

Meta-analysis of all studies showed a significantly better pCR, T downstaging, $\mathrm{N}$ downstaging, when radiation therapy is combined with capecitabine instead of 5-FU for the neoadjuvant treatment of LARC. As far as the adverse events were concerned, there appeared to be higher rate of handfoot syndrome and lower rate of lowered leucocytes in the capecitabine arm. However, the type of surgical resection and other adverse events (lowered hemoglobin, lowered platelets, nausea, vomiting, diarrhea, mucositis, and radiation dermatitis) were not statistically different between these two regimens. Since the data on diarrhea, radiation dermatitis were different between the pooled trials; they should be interpreted with caution.

Only four trials mentioned long-term survivorship; interestingly, in all studies, capecitabine showed reduction in distant metastases and noninferiority in both DFS and OS, suggesting greater systemic efficacy than with 5-FU, in accordance with the X-ACT study comparing capecitabine with bolus fluorouracil plus folinic acid for adjuvant treatment of stage III colon cancer. This could be partly explained by the higher $\mathrm{pCR}$ rates of capecitabine group. As proved by other trials [25], patients with a pCR after chemoradiation have a significantly better long-term outcome than do those with residual disease, although this could be influenced by adjuvant treatment; additional evidence will be required to assess the long-term prognosis of capecitabine and 5-FU in the preoperative combined-modality therapy of LARC.

Hand-foot syndrome is predominant in capecitabine treatment group but could be easily managed with vitamin B6 administration and supportive care without interrupting the RT schedule. Interestingly, in the trial of Hofheinz et al., those patients who developed HFS enjoyed better DFS and overall survival than those who did not develop in both capecitabine group and the overall study population [12].

Our study had several limitations, and the efficiency of this meta-analysis might have been affected by several factors. First of all, because of the possibility that not all relevant studies were identified by computerized searching, it was necessary to combine the electronic material with manual research and personal contact with authors. Unfortunately, efforts on this were not very productive. Secondly, only two RCTs were included in this meta-analysis, and the number of patients in NSABP-R-04 accounts for almost one-half; the small sample sizes of the retrospective trials included might affect the conclusion. Thirdly, the usage of oxaliplatin may also influence the pCR rate in the NSABP-R-04 trial. Finally, varying doses and application of capecitabine and 5FU might influence the results.
In conclusion, as a convenient oral fluoropyrimidine, capecitabine seemed to be at least as good as continuous 5-FU and was an acceptable alternative in the neoadjuvant chemoradiation treatment of patients with LARC. It was associated with improvement tumor response rate and favourably low toxicity with the exception of HFS. Based on these results, we would recommend the use of capecitabine combined with pelvic radiation as a neoadjuvant treatment for LARC.

\section{Competing Interests}

The authors have declared no competing interests.

\section{Authors' Contributions}

Guo-Chen Liu, Jun-Ping Yan, and Qing He contributed equally to this work.

\section{Acknowledgments}

The authors thank Kun Zhao for his input on text modification. This work was supported by Nature Science Foundation of China [Grant no. 81101591].

\section{References}

[1] R. Sauer, H. Becker, W. Hohenberger et al., "Preoperative versus postoperative chemoradiotherapy for rectal cancer," The New England Journal of Medicine, vol. 351, no. 17, pp. 1731-1810, 2004.

[2] T. A. Rich, J. M. Skibber, J. A. Ajani et al., "Preoperative infusional chemoradiation therapy for Stage T3 rectal cancer," International Journal of Radiation Oncology, Biology, Physics, vol. 32, no. 4, pp. 1025-1029, 1995.

[3] N. A. Janjan, C. N. Crane, B. W. Feig et al., "Prospective trial of preoperative concomitant boost radiotherapy with continuous infusion 5-fluorouracil for locally advanced rectal cancer," International Journal of Radiation Oncology Biology Physics, vol. 47, no. 3, pp. 713-718, 2000.

[4] C. H. Crane, J. M. Skibber, E. H. Birnbaum et al., "The addition of continuous infusion 5-FU to preoperative radiation therapy increases tumor response, leading to increased sphincter preservation in locally advanced rectal cancer," International Journal of Radiation Oncology Biology Physics, vol. 57, no. 1, pp. 84-89, 2003.

[5] M. J. O'Connell, J. A. Martenson, H. S. Wieand et al., "Improving adjuvant therapy for rectal cancer by combining protractedinfusion fluorouracil with radiation therapy after curative surgery," The New England Journal of Medicine, vol. 331, no. 8, pp. 502-507, 1994.

[6] H. P. Kalofonos, A. Bamias, A. Koutras et al., "A randomised phase III trial of adjuvant radio-chemotherapy comparing Irinotecan, 5FU and Leucovorin to 5FU and Leucovorin in patients with rectal cancer: a Hellenic Cooperative Oncology Group Study," European Journal of Cancer, vol. 44, no. 12, pp. 1693-1700, 2008.

[7] I. Di Carlo, E. Pulvirenti, M. Mannino, and A. Toro, "Increased use of percutaneous technique for totally implantable venous access devices. Is it real progress? A 27-year comprehensive review on early complications," Annals of Surgical Oncology, vol. 17, no. 6, pp. 1649-1656, 2010. 
[8] R. J. Mayer, "Should capecitabine replace infusional fluorouracil and leucovorin when combined with oxaliplatin in metastatic colorectal cancer?" Journal of Clinical Oncology, vol. 25, no. 27, pp. 4165-4167, 2007.

[9] E. Van Cutsem, M. Findlay, B. Osterwalder et al., "Capecitabine, an oral fluoropyrimidine carbamate with substantial activity in advanced colorectal cancer: results of a randomized phase II study," Journal of Clinical Oncology, vol. 18, no. 6, pp. 1337-1345, 2000.

[10] P. M. Hoff, R. Ansari, G. Batist et al., "Comparison of oral capecitabine versus intravenous fluorouracil plus leucovorin as first-line treatment in 605 patients with metastatic colorectal cancer: results of a randomized phase III study," Journal of Clinical Oncology, vol. 19, no. 8, pp. 2282-2292, 2001.

[11] J. Dunst, T. Reese, T. Sutter et al., "Phase I trial evaluating the concurrent combination of radiotherapy and capecitabine in rectal cancer," Journal of Clinical Oncology, vol. 20, no. 19, pp. 3983-3991, 2002.

[12] R.-D. Hofheinz, F. Wenz, S. Post et al., "Chemoradiotherapy with capecitabine versus fluorouracil for locally advanced rectal cancer: a randomised, multicentre, non-inferiority, phase 3 trial," The Lancet Oncology, vol. 13, no. 6, pp. 579-588, 2012.

[13] M. J. O'Connell, L. H. Colangelo, R. W. Beart et al., "Capecitabine and oxaliplatin in the preoperative multimodality treatment of rectal cancer: surgical end points from national surgical adjuvant breast and bowel project trial R-04," Journal of Clinical Oncology, vol. 32, no. 18, pp. 1927-1934, 2014.

[14] A. K. Chan, A. O. Wong, and D. A. Jenken, "Preoperative capecitabine and pelvic radiation in locally advanced rectal cancer-is it equivalent to 5-FU infusion plus leucovorin and radiotherapy?" International Journal of Radiation Oncology, Biology, Physics, vol. 76, no. 5, pp. 1413-1419, 2010.

[15] C.-F. Chen, M.-Y. Huang, C.-J. Huang et al., "A observational study of the efficacy and safety of capecitabine versus bolus infusional 5-fluorouracil in pre-operative chemoradiotherapy for locally advanced rectal cancer," International Journal of Colorectal Disease, vol. 27, no. 6, pp. 727-736, 2012.

[16] P. Das, E. H. Lin, S. Bhatia et al., "Preoperative chemoradiotherapy with capecitabine versus protracted infusion 5-fluorouracil for rectal cancer: a matched-pair analysis," International Journal of Radiation Oncology Biology Physics, vol. 66, no. 5, pp. 13781383, 2006.

[17] D. Y. Kim, K. H. Jung, T. H. Kim et al., "Comparison of 5-fluorouracil/leucovorin and capecitabine in preoperative chemoradiotherapy for locally advanced rectal cancer," International Journal of Radiation Oncology Biology Physics, vol. 67, no. 2, pp. 378-384, 2007.

[18] J.-S. Kim, J.-S. Kim, M.-J. Cho, W.-H. Yoon, and K.-S. Song, "Comparison of the efficacy of oral capecitabine versus bolus 5-FU in preoperative radiotherapy of locally advanced rectal cancer," Journal of Korean Medical Science, vol. 21, no. 1, pp. 5257, 2006.

[19] V. S. Ramani, A. Sun Myint, A. Montazeri, and H. Wong, "Preoperative chemoradiotherapy for rectal cancer: a comparison between intravenous 5-fluorouracil and oral capecitabine," Colorectal Disease, vol. 12, no. 2, pp. 37-46, 2010.

[20] R. Yerushalmi, E. Idelevich, Y. Dror et al., "Preoperative chemoradiation in rectal cancer: retrospective comparison between capecitabine and continuous infusion of 5-fluorouracil," Journal of Surgical Oncology, vol. 93, no. 7, pp. 529-533, 2006.
[21] D. L. Demets, "Methods for combining randomized clinical trials: strengths and limitations," Statistics in Medicine, vol. 6, no. 3, pp. 341-348, 1987.

[22] R. DerSimonian and N. Laird, "Meta-analysis in clinical trials," Controlled Clinical Trials, vol. 7, no. 3, pp. 177-188, 1986.

[23] M. Egger, G. D. Smith, M. Schneider, and C. Minder, "Bias in meta-analysis detected by a simple, graphical test," The British Medical Journal, vol. 315, no. 7109, pp. 629-634, 1997.

[24] P. Piedbois, P. Rougier, M. Buyse et al., "Efficacy of intravenous continuous infusion of fluorouracil compared with bolus administration in advanced colorectal cancer," Journal of Clinical Oncology, vol. 16, pp. 301-308, 1998.

[25] M. Maas, P. J. Nelemans, V. Valentini et al., "Long-term outcome in patients with a pathological complete response after chemoradiation for rectal cancer: a pooled analysis of individual patient data," The Lancet Oncology, vol. 11, no. 9, pp. 835-844, 2010. 


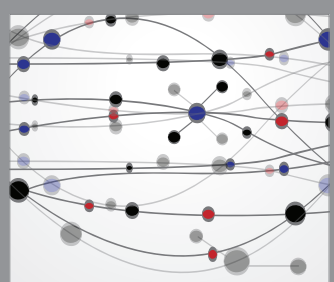

The Scientific World Journal
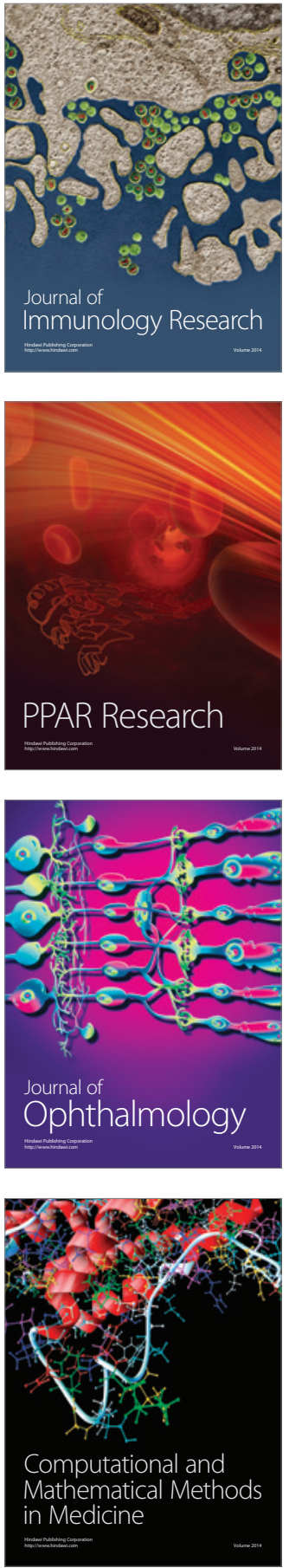

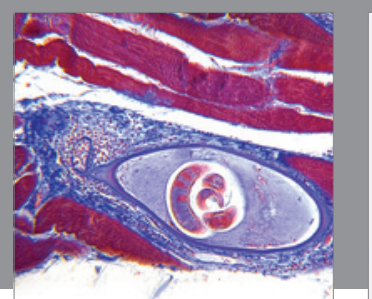

Gastroenterology Research and Practice

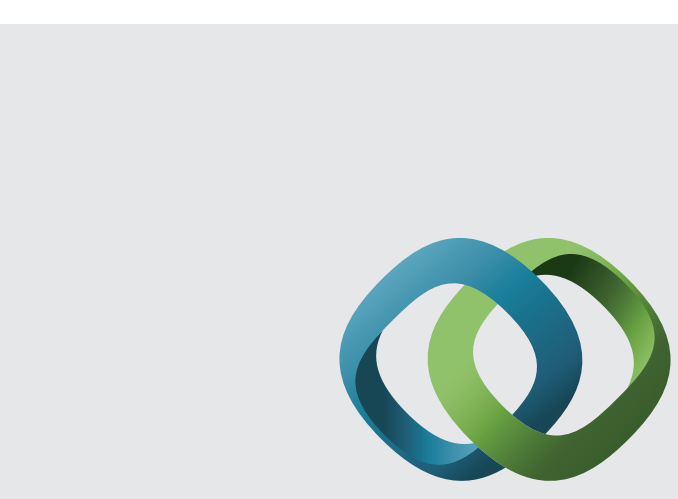

\section{Hindawi}

Submit your manuscripts at

http://www.hindawi.com
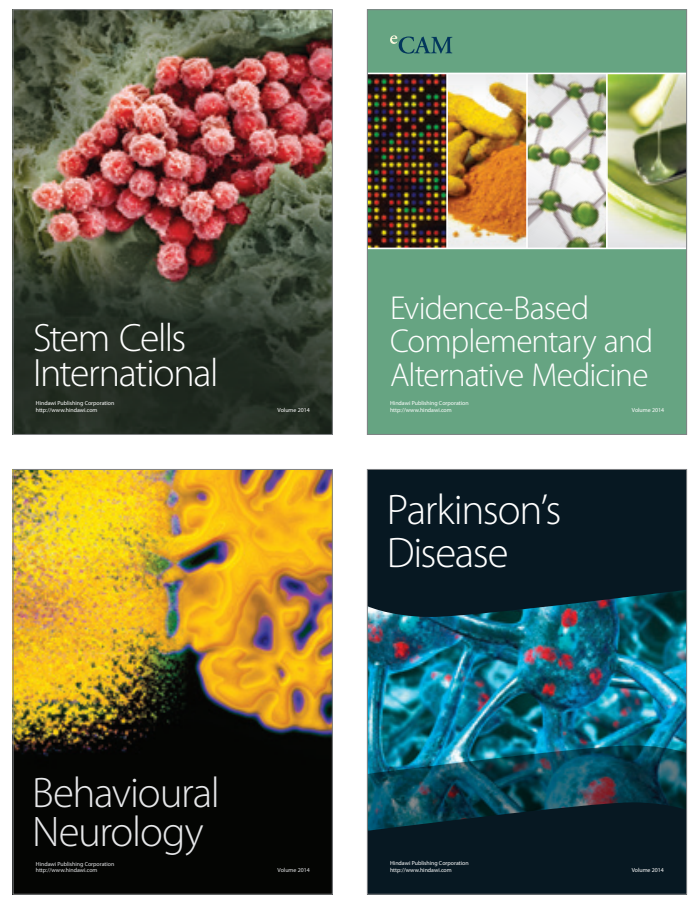
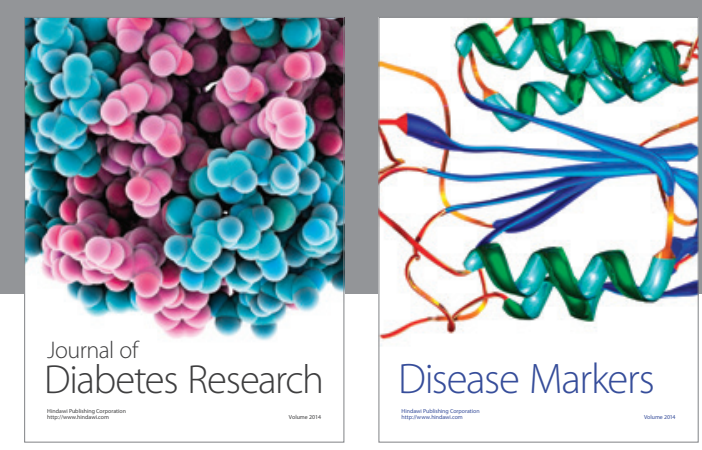

Disease Markers
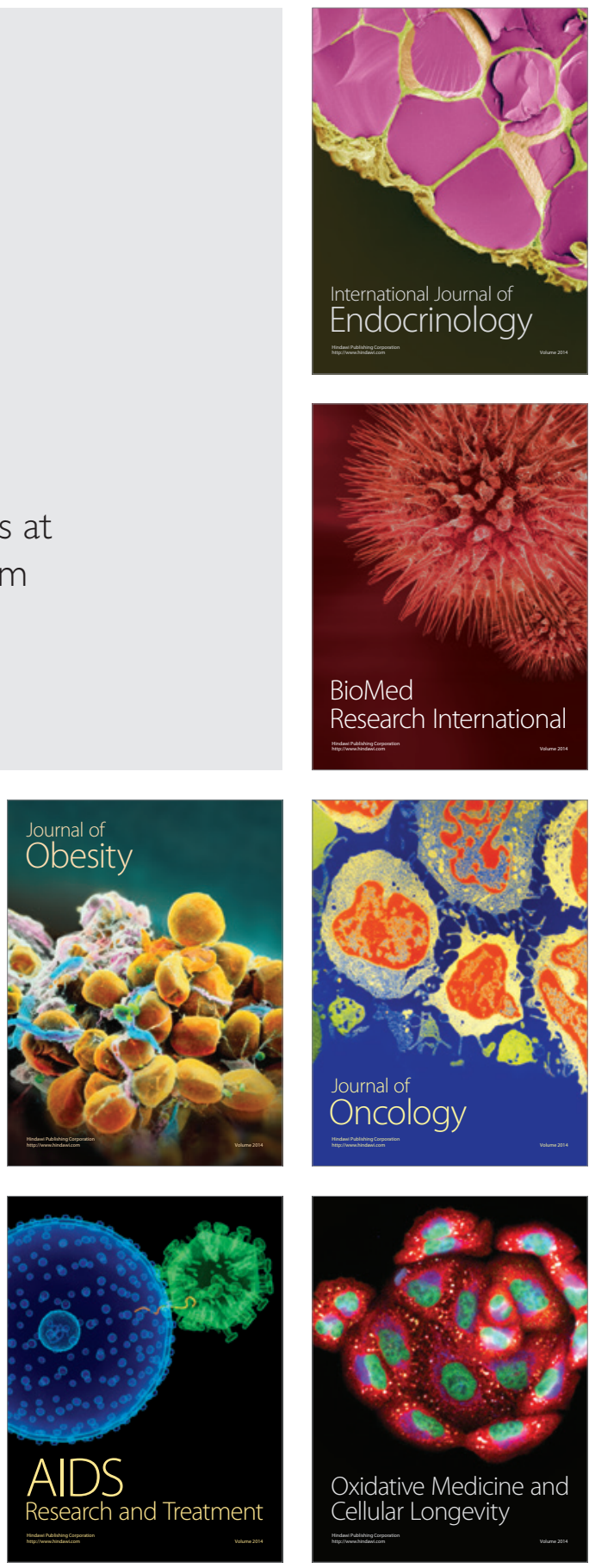